\title{
QUAND LES ACTEURS PRENNENT LA PLUME. PRONUNCIARSE DANS LE VENEZUELA INDÉPENDANT $(1828-1858)^{1}$ \\ (1) Veronique Hébrard ${ }^{23}$
}

This article aims to analyze the dynamics of socio-political mobilization as manifested in 19th century Venezuela through a practice little studied in this perspective: the pronouncement. Almost always considered in its armed dimension, it should be mentioned that it also means, through the break of the pact, an interpellation of government agents, at the local and/or national level, that is, the expression of a collective will in the face of a specific situation. On this occasion hundreds of texts are written, debated and

1 Cet article est en partie né d'une réflexion comparative sur le pétitionnement entre les États-Unis et l'Amérique latine menée avec Romain Huret (EHESS) et présentée dans le séminaire L'État dans les Amériques de I'UMR 8168 Mondes Américains (EHESS).

2 Université Lille. Lille - France.

3 Véronique Hébrard est professeure d'histoire latino-américaine contemporaine à l'Université de Lille. Elle est spécialiste de l'histoire politique du Venezuela au XIXe siècle et plus particulièrement des processus de mobilisation et de politisation des populations civiles en temps de guerre. Entre autres choses, Elle a publié La Venezuela independiente. Una nación de discurso (1808-1830), Madrid, Vervuert/lberoamericana, 2012, 628 p ; « Ciudades leales, ciudades patriotas. Guerra de independencia y identidades urbanas (Venezuela, siglo XIX)», Tiempo y Espacio (Caracas), $n^{\circ}$ 63, enero-junio 2015, pp. 163-186; « Justicia'excepcional'y lógicas de radicalización durante la primera Pacificación de Venezuela (1812-1814) », Revista de Indias (Madrid), número especial coordinado por Flavia Macias y Marta Irurozqui, 2016, vol. LXXVI, n. 266, pp. 17-50. Il s'intéresse également aux archives des américanistes européens : Véronique Hébrard (dir.), Véronique Hébrard (dir.), Por una concepción atlántica del americanismo. En los pasos de François Chevalier, Paris, Editions des Archives Contemporaines, 2013. ; Véronique Hébrard (dir.), Sur les traces d'un mexicaniste français. Constitution et analyse du fonds François Chevalier. Paris : Karthala, 2005. 
signed by social actors, even those who do not enjoy the status of citizen, and in most cases published in the following months. However, although it has no legality, the study at the same time of the practices, but also of the vocabulary and sociability that they generate, allows to show at the same time their real role in the political debate, the level of politicization of an important spectrum of social actors, but also its legitimacy. Considering it then as an observatory of the phenomena of mobilization and politicization as elaborated during the first decades of national construction, we will study two important campaigns of pronouncements/votes that affect Venezuela, in 1827 to ask Bolivar to exercise extraordinary powers and in 1858 in the context of the Impeachment of President JoséTadeo Monagas.

Venezuela - pronunciamiento - petition - politization $-19^{\text {th }}$ Century. 


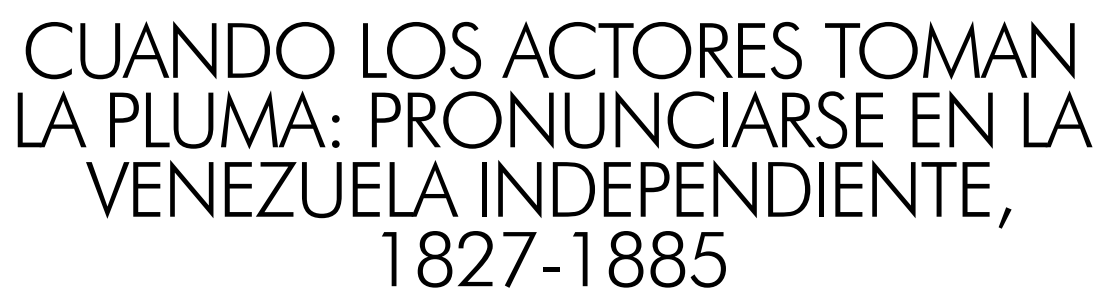

Resumen

Este artículo tiene como objetivo analizar las dinámicas de movilización socio-políticas tales como se manifiestan en la Venezuela del siglo XIX a través de una práctica poco estudiada en esta perspectiva: el pronunciamiento.

Casi siempre considerado en su dimensión armada, cabe mencionar que significa también, mediante la ruptura del pacto que supone, una interpelación de los agentes del gobierno, a nivel local y/o nacional, es decir la expresión de una voluntad colectiva frente a una situación específica.

En esta ocasiones centenares de textos son redactados, debatidos y firmados por actores sociales, incluso aquellos que no gozan del estatuto de ciudadano, y en la mayoría de los casos publicados en los meses siguientes. Ahora bien, y aunque no tenga ninguna legalidad, el estudio a la vez de las prácticas, pero también del vocabulario y de la sociabilidad que generan, permite mostrar a la vez su papel real en el debate político, el nivel de politización de un importanteespectro deactores sociales, pero también su legitimidad frente a los representantes del Estado. Considerándoloentoncescomo un observatorio delosfenómenos de movilización y politización tal como se elaboran durante las primeras décadas de la construcción nacional, estudiaremos dos importantes campañas de pronunciamientos/votos que afectan a Venezuela, en 1827 para pedir a Bolívar que ejerce poderes extraordinarios y en 1858 en el marco de la destitución del presidente José Tadeo Monagas.

\section{Palabras clave}

Venezuela - pronunciamiento - petición - politización - siglo XIX. 
et article a pour objectif d'étudier la façon dont une large palette d'acteurs sociopolitiques du XIXème siècle vénézuélien s'est emparée de la plume, en particulier par le biais du pronunciamiento, pour interpeller les agents et représentants de l'Etat. Or, cette pratique plus souvent appréhendée dans sa dimension armée, est très rarement étudiée dans ce qu'elle signifie en termes de rupture « pacifique » du pacte et d'interpellation écrite des autorités au niveau local et/ou national. C'est en cela d'ailleurs qu'elle se rapproche de ce que l'on nomme plus génériquement le pétitionnement, tout en s'en distinguant sur certains aspects que je m'attacherai de mettre au jour.

Or, un tel recours à la plume ne manque pas d'interpeller à son tour I'historien dans la mesure où ces écrits, longtemps délaissés ou traités defaçon anecdotique ou commedesensembles indifférenciés, s'avèrent d'une grande valeur heuristique pour étudier la façon dont de larges secteurs de la population se mobilisent autour d'une «cause » mais aussi dont ils conçoivent le rôle et les attributions des institutions politiques et de leurs représentants. Ils constituent en cela un miroir des cultures et représentations politiques. Surtout en ce qui concerne les secteurs dits populaires et/ou pour le moins éloignés de l'écrit.

Il s'agira donc ici de mettre en évidence non seulement les dynamiques qui conduisent les acteurs à prendre la plume mais aussi les mécanismes qui président à l'élaboration de ces documents, qu'il s'agisse de pronunciamiento, votos, representaciones ou actas (de l'écriture du texte servant de matrice, à sa circulation, en passant par les modalités mises en œuvre pour médiatiser son contenu, en débattre et pour collecter les signatures).

Or, à plusieurs reprises au XIXème, des campagnes de pronunciamientos/votos s'organisent, à l'occasion desquelles des centaines de textes sont rédigés, débattus, signés puis, dans certains cas, réunis et publiés au cours des semaines ou mois qui suivent. A partir de l'étude des pratiques et formes de sociabilités qui les accompagnent, mais aussi des formes de sociabilité qu'ils occasionnent, on peut mettre au jour la fonction qui est la sienne dans le débat politique et public, les 
modalités d'énonciation de cette opinion particulière et par la même de mesurer les formes de politisation d'un important spectre d'acteurs sociaux.

Enles considérant dès lors comme de véritables observatoires des phénomènes de mobilisation et modes de politisation tels qu'ils se structurent et s'énoncent dans ces premières décennies du XIXème siècle, je m'intéresserai ici à deux de ces campagnes de pronunciamientos qui se déploient au Venezuela, en 1827 pour la première, et destinée en tout premier lieu à demander à Bolívar qu'il exerce des pouvoirs extraordinaires afin de sauver la République de Colombie ; en 1858 pour la seconde dans le cadre de la destitution du président JoséTadeo Monagas suiteà coup de force armémenépar Julián Castro.

En les considérant ainsi dans leur matérialité et leur contenu, ces deux campagnes de pronunciamientos peuvent permettre de comprendre ce que ces écrits singuliers nous disent des interactions entre les acteurs sociaux mobilisés, l'Etat et ses agents, dans la mesure où cette articulation permet de réfléchir aux liens entre la société et les formes étatiques d'autorité ${ }^{4}$, mais aussi à des formes d'agentivité peut questionnées par l'historiographie.

Après un tour d'horizon historiographique, je m'attacherai, à partir d'exemples concrets, à rendre compte du cadre légal dans lequel ces écrits s'inscrivent, puis aux finalités de ces deux formes d'écrits avant de décrire ce qu'ils nous disent sur les acteurs eux-mêmes.

\section{De l'oubli au renouveau: un bilan historiographique}

De façon générale, et en dépit de l'importance de la pratique pétitionnaire au sens large du terme, au XIXe siècle, les études sont

4 Comme l'indique très justement Agnès Benoît dans une publication récente, les pétitions se situent en effet « au centre des rapports entre société et autorité ». BENOÎT, Agnès. L'appel au pouvoir. Les pétitions aux Parlements en France et au Royaume-Uni (1814-1848). Rennes : Presses Universitaires de Rennes, 2018. p.11. 
finalement peu nombreuses, un paradoxe au regard du dynamisme de l'« intellectualisme pétitionnaire », bien analysé par l'historienne Madeleine Rebérioux pour le cas français ${ }^{5}$. Al'exception des moments révolutionnaires de la fin du XVIIlème siècle européen, le pétitionnement est globalement négligé par les historiens, les sociologues et les politologues, alors que des sources volumineuses existent aussi bien à l'échelle locale que nationale. Dans les écrits, l'âge d'or de la pétition semble coïncider avec l'ère des Révolutions, et la richesse de l'historiographie française sur les cahiers de doléances et l'explosion du pétitionnement révèle bien la coïncidence entre essor du pétitionnement et démocratisation des sociétés occidentales ${ }^{6}$. La création d'un espace public ouvre la voie à une transformation du pétitionnement, qui existait dès les temps médiévaux.

Etudiant la Grande-Bretagne des $17^{\text {ème }}$ et $18^{\text {ème }}$ siècles, le sociologue David Zaret a mis en avant l'importance de la pétition dans la construction d'une sphère publique. Selon lui, le pétitionnement a été négligé par les historiens de la culture, plus sensibles à d'autres formes d'imprimé. La pétition permet pourtant d'entendre les voix d'individus qui ont peu accès aux espaces de sociabilité éclairés. Au lendemain des révolutions atlantiques, les pétitions ne disparaissent pas, mais acquièrent d'autres contours et fonctions dans le même temps qu'elles sont recodifiées par le droit dans le cadre des institutions représentatives dès lors que se produit une dynamique de politisation de ces formes anciennes d'interpellation et un usage de plus

5 RÉBÉRIOUX, Madeleine. Pétitionner ». In : RÉBÉRIOUX, Madeleine (dir.). Pour que vive l'histoire. Écrits. Paris : Belin, 2017. pp.449-455.

6 On peut mentionner : WAHNICH, Sophie. La pétition : une politisation de la plainte, 1789-1792. Annales de l'Est, 2007-2. pp.71-87 ; CHRISTIN, Olivier ; FOA, Jérémie (ed.). Pétitions et suppliques, Annales de l'Est, Vol. 57, n. 2, 2007. DIONNET, Jean-Pierre. Le droit de pétition durant la Restauration (1814-1830) : contribution à I'histoire socio-politique française du XIXème siècle, thèse pour le doctorat en histoire du droit, université de Poitiers, 2001 ; CONTAMIN, Jean-Gabriel. Contribution à une sociologie des usages pluriels des formes de mobilisation: l'exemple de la pétition en France, thèse pour le doctorat en science politique, université Paris 1, dir. Michel Offerlé, 2001 ;MÉROT, Catherine (dir.). L'individu face au pouvoir : les pétitions aux assemblées parlementaires. Revue administrative, $61^{\text {ème }}$ année, numéro spécial, 2008. 
en plus collectif, suscitant la crainte des autorités et leur souci de légiférer afin d'en encadrer le recours.

Il en va de même dans l'espace ibéro-américain où, au même titre que la manifestation, la pétition « illustre la pluralité des formes de l'expression politique, en même temps que les tentatives récurrentes du pouvoir pour les canaliser ${ }^{7}$, voire les désinstitutionnaliser. Ces appels au pouvoir, qui participent de la constitution d'une sphère publique moderne et démocratique, contribuent également aux dynamiques d'incorporation (par leur usage et/ou leur lecture et écoute) du caractère "représentatif » des nouveaux régimes, de la politisation des acteurs et, par conséquent aussi, d'une injonction de la part de ces derniers, de reconnaissance d'une plus large palette d'acteurs que le seul corps politique des citoyens, dans des contextes où celui-ci demeurent restreint, même si cette corrélation n'est pas toujours vérifiée ${ }^{8}$. L'analyse de ces phénomènes d'interpellation ne peut donc s'opérer sans leur mise en rapport d'une part avec les contours de la citoyenneté et, d'autre part, avec la façon dont le droit de pétition est, ou non, reconnu dans les textes législatifs en vigueur.

Concernant l'Amérique latine, cette pratique, pourtant omniprésente dans les représentations sur le fonctionnement (voire dysfonctionnement) du politique dans I'Amérique latine du XIXème siècle, est paradoxalement un angle mort dans I'historiographie sur le politique et sur les formes de mobilisation et politisation des acteurs.

Notons tout d'abord qu'il existe peu d'études approfondies sur les pétitions en tant que telles. Fait exception le Mexique avec les travaux de Romana Falcón, tout particulièrement sur ce qu'elle appelle « l'art de la pétition ${ }^{9}$, phénomène qui vient s'incruster dans une

7 VERDO, Geneviève. Pierre Rosanvallon, archéologue de la démocratie. Revue historique, 2002, n. 3, p.714.

8 C'est ce qu'indique A. Benoît à propos des différences constatées entre la France et l'Angleterre quant au recours à la pétition. Il est plus important dans ce dernier pays alors que c'est en France que le suffrage est le plus restreint. BENOÎT, Agnès. Op. Cit., p.21-22.

9 FALCÓN, Romana. El arte de la petición: rituales de obediencia y negociación, México, segunda mitad del siglo XIX. Hispanic American Historical Review, vol. 86, n. 3, 2006, p.467-500. 
pratique ancienne d'interpellation à l'initiative tout particulièrement des communautés indigènes qui dénonce les politiques de démantèlement des terres communales et se renouvelle dans les années 1830/1840, puis en 1856-1857 et lors de la chute de Maximilien. Cette pratique est à tel point mobilisée qu'un autre historien du Mexique, Antonio Annino, a pu dire que « dans les années trente et quarante une grande vague de pétitions de pueblos en défense de leurs terres a inondé aussi bien les congrès des états que centraux. La quantité de pétitions fut telle que les congressistes ont dû discuter sérieusement, bien que sans succès, sur la façon de limiter ce phénomène ", témoignant ainsi de la persistance, de la part des communautés, de cette façon de «'se représenter'face aux nouveaux pouvoirs » ${ }^{10}$. François-Xavier Guerra s'y est lui aussi intéressé, en insistant surtout sur les pronunciamientos qu'il considère comme «l'une des pratiques politiques les plus importantes du XIXème siècle » qui « suppose des causes structurelles renvoyant à un certain type de régime, d'imaginaires et de comportements politiques ${ }^{11}$. Toutefois, il l'analyse pour l'essentiel sous l'angle du coup de force initié par des militaires, qui rédigent un « plan », texte initial, suivi d'actes d'adhésion ou de rejet, dans lesquels des individus ou des corporations de toutes sortes se "prononcent », voire se " déprononcent ${ }^{12}$. On y trouve une diversité extraordinaire d'acteurs de tout type en effet, certains individuels mais surtout collectifs, civils, militaires ou ecclésiastiques, du haut en bas de l'échelle sociale (congrès des Etats, villes, pueblos de indios, barrios, garnisons, milices civiles, corps de métiers ou pères de famille), constitués pour la circonstance. Néanmoins, cette primauté de l'ini-

10 ANNINO, Antonio. Pueblos, liberalismo y nación en México. In ANNINO, Antonio ; GUERRA, François-Xavier (dir.). Inventando la nación: Iberoamérica siglo XIX. México, Fondo de Cultura Económica, 2003, p.428.

11 GUERRA, François-Xavier. El pronunciamiento en México: prácticas e imaginarios. Trace, n. 37, juin 2000, p.15.

12 Acta de despronunciamiento de ciudad Guerrero, 17 de mayo de 1840, Guerrero, Tamaulipas. http://arts.st-andrews.ac.uk/pronunciamientos/database/index.php?id=184. 
tiative militaire du pronunciamiento et la primauté qu'on lui accorde ${ }^{13}$, est loin d'être sa caractéristique première comme je vais m'efforcer de le montrer. De plus, dans certains travaux la pétition et le pronunciamiento sont parfois pris l'un pour l'autre, et l'on trouve parfois des références à des "pétitions » alors que la source mentionnée porte dans son titre, "pronunciamiento ». Or il me semble qu'il ne s'agit pas là d'une simple question sémantique, et que cette imprécision historiographique fait l'impasse sur une praxis singulière au sens où recourir à un terme plutôt qu'un autre, de même que la présence plus importante de l'une ou l'autre de ces formes d'interpellation n'obéit pas aux mêmes logiques.

De ce point de vue, ce sont surtout les travaux que Marie-Danielle Demélas a consacrés à la Bolivie (et plus marginalement au Pérou) et aux nombreux pronunciamientos rédigés au XIXème siècle dans ces sociétés extrêmement excluantes en termes de citoyenneté, qui ont le plus contribué à une approche renouvelée de cette pratique qu'elle considère comme une résurgence du pactisme ${ }^{14}$. En effet, ici aussi les communautés indigènes ont tout particulièrement mobilisé cette arme de papier pour faire valoir leurs droits et exprimer leur revendication, au point d'en renouveler l'usage au XXlème siècle dans la Bolivie d'Evo Morales ${ }^{15}$. Ailleurs, on trouve bien sûr quelques travaux ponctuels, en particulier sur l'Argentine et la Colombie, à l'instar des travaux de R. Garcilazo pour la première ${ }^{16}$ et de Clément Thibaud et

13 Voir l'article de FALLAS SANTANA, Carmen María. La voluntad de la Nación y la regeneración política: Los pronunciamientos militares de 1859, 1868 y 1870 en Costa Rica, Diálogos Revista Electrónica de Historia, Vol. 9, n. 2 Agosto 2008 - Febrero 2009, p.54-76.

14 DEMÉLAS-BOHY, Marie-Danielle. Pactismo y constitucionalismo en los Andes. In. ANNINO, Antonio ; CASTRO LEIVA, Luis ; GUERRA, François-Xavier (dir.), De los Imperios a las Naciones : Iberoamérica. Zaragoza: IberCaja, 1994, p.495-510. Voir en particulier l'item intitulé 'El pronunciamiento como resurgimiento del pactismo', p.499-503.

15 DEMÉLAS, Marie-Danielle. Retour sur la pratique du pronunciamiento (1820-2013). Problèmes d'Amérique latine, 2014/1, n. 91, p.41-51.

16 GARCILAZO, Romina. Recurrir a las autoridades por una justa causa. El derecho de petición en la ciudad de Rosario (Argentina), 1883/84-1890. Temas Americanistas, n. 33, 2014,pp.130-152. 
Maria-Teresa Calderón pour la seconde ${ }^{17}$. Sur le Venezuela, hormis l'article de Thibaud et mes propres travaux ${ }^{18}$, aucune étude d'envergure n'a été entreprise alors que le pays connaît plusieurs campagnes de pronunciamientos de grande ampleur au XIXème siècle, outre des formes d'interpellations plus ponctuelles.

Toutefois, et en dépit de l'apport de ces différentes études, aucune ne s'est intéressée spécifiquement à cette pratique en tant que telle, et moins encore au pronunciamiento dans sa matérialité (dans sa morphologieet physionomie).Cequiestd'autant plus surprenantdès lors que ces pratiques constituent l'arrière-plan de nombreux travaux sur le politique en Amérique latine, mais dépourvus d'une étude des textes proprement dits alors que leur valeur heuristique est double. II nous renseigne en effet sur les attentes des acteurs mobilisés (soient-ils rédacteurs ou seulement signataires), sur leur profil, mais aussi sur les modes d'énonciation du politique, d'appropriation du langage de la modernité et son éventuelle resémantisation.

\section{Pétition et pronunciamiento : statut juridique et morphologie}

Avant de considérer le cadre légal dans lequel ces formes d'interpellations s'inscrivent, une précision s'impose qui, de mon point de vue, n'est pas sémantique. A savoir que dans le Venezuela du XIXème siècle, on pétitionne très peu, tandis que l'on se « prononce », émet

17 THIBAUD, Clément. Entre les cités et l'État. Caudillos et pronunciamientos en Grande-Colombie. Genèses, 2006/1, n. 62, p.5-26. CALDERÓN, María Teresa. 1825-1832, Crisis y disolución de la unión colombiana. In: PORTILLO, José María ; CAGIAO VILA, Pilar (dir.). Entre imperio y naciones: Iberoamérica y el Caribe en torno a 1810. Santiago de Compostela: Universidad de Santiago de Compostela, 2012, p.215-244.

18 HÉBRARD, Véronique. El recurso al poder personal y autoritario ». In HÉBRARD, Véronique. La Venezuela independiente. Una nación de discurso (1808-1830). Madrid:Vervuert/lberoamicana, 2012, p.446-548 ; HÉBRARD, Véronique. Quand la parole est collective. In. Véronique HÉBRARD, La Faction de la Sierra. Un apprentissage du politique entre engagement et contrainte. Venezuela, 1858-1859. Inédit d'HDR. Université Paris Ouest Nanterre La Défense, 2013, p.249-255. HAL Id : tel-01180313, version 1. 
des Votos, souvent sur les affaires politiques du pays. Or, I'analyse de la législation peut justement être une entrée pertinente pour décoder ce constat. Que nous dit le droit sur la période étudiée et pour les deuxmomentsoù seproduisentles campagnes de pronunciamientos retenues pour cette étude?

De façon générale, et la dynamique est la même pour l'ensemble des pays de l'Amérique espagnole, si le droit de pétition est reconnu, il est circonscrit aux seules « plaintes » et maux dont les habitants pourraient souffrir et pour lesquels ils peuvent adresser des pétitions aux représentants locaux ou centraux. II est en revanche interdit, dans la constitution de 1811, de rédiger des pétitions « au nom du Peuple, et de «s'attribuer la qualification de Peuple Souverain ${ }^{19}$.

En revanche, nulle référence à ce droit dans les Constitutions d'Angostura (1819) et de Cucutá (1821), même si le droit « d'exprimer ses pensées et opinions oralement, par écrit, ou de toute autre manière, et le premier et le bien le plus précieux de l'homme en société » est reconnu. Les limites fixées sont celles habituellement énoncées puisqu'il est rappelé que « chacun est responsable de ses écrits et paroles, et que des peines appropriées sont prises à l'encontre de ceux qui l'exercent de façon licencieuse au préjudice de la tranquillité publique, des bonnes coutumes, de vie, de l'honneur, du respect et de la propriété individuelle $»^{20}$. Toutefois, l'article suivant, qui limite la " liberté de faire valoir ses droits, à condition que ce soit fait individuellement » reconnaît que « pour des affaires communes à plusieurs individus, ou d'intérêt général, on peut faire une représentation par corps, à condition que ce soit par écrit $»^{21}$.

Dans la Constitution de Cucutá, la liberté de pensée et d'opinion est réaffirmée en des termes clairs, puisqu'il est indiqué que « tous les Colombiens ont le droit d'écrire, d'imprimer et de publier librement

19 Constitución federal para los Estados de Venezuela de 1811, Sección segunda. Derechos del hombre en sociedad, Art. 182 et 215.

20 Constitución de 1819, Título 1. Derechos y deberes del hombre y del ciudadano, Art. 4.

21 Ibid. Art. 5. 
leurs pensées et opinions, sans besoind'un examen, d'une révision ou quelque censure que ce soit préalablement à la publication ». Mais, « ceux qui abusent de cette précieuse faculté endureront les peines dont ils seront passibles conformément à la loi » ${ }^{22}$. Dans les trois constitutions de 1830 et 1857 et 1864 , on accorde le droit de « représenter par écrit » au Congrès et autres pouvoirs constitués, mais là encore il est fait interdiction de faire « des pétitions au nom du peuple, ou de s'arroger le qualificatif de peuple», et lorsque des pétitions sont rédigées par "de nombreux individus ", " tous seront responsables de la vérité des faits et les cinq premiers signataires responsables de l'identité de toutes les signatures ${ }^{23}$.

Toutefois et en dépit d'un large droit d'expression, nulle référence directe au droit de pétition et à ses restrictions comme dans les constitutions précédentes. Ce qui néanmoins ne manque pas l'interpeller. Dans les constitutions suivantes, le droit de pétition (à l'instar des autres droits et libertés) tend à s'assouplir et surtout à reconnaître le caractère collectif de celle-ci, tant redoutéeauparavant, de mêmeque sa politisation de ces formes anciennes d'interpellation qui caractérise la forme pétitionnaire post-révolutionnaire ${ }^{24}$.

Bien sûr, on retrouve, dans la pratique pétitionnaire codifiée dans la plupart des constitutions, des éléments très semblables à la forme prise par le pronunciamiento (tout particulièrement le fait qu'il s'agisse de documents écrits par un collectif et comportant des signatures). Cependant, le sens qu'ils revêtent et leur fonction sur la scène politique obéissent à des moyens et des fins distincts. Et surtout, le pronunciamiento n'est l'objet d'aucune codification : rien dans les constitutions, pas plus que dans les lois et décrets ne fait référence au

22 Constitución de 1821. Título VIII. Disposiciones générales, Art. 156.

23 Constitución de 1830, Título 26. Disposiciones générales, Art. 19; Constitución de 1857, Título XX. De las garantías, Art. 115 et 116 ; Constitución de 1864, Título III. Garantía de los venezolanos, Art. 14.

24 Comme l'a bien montré Sophie Wahnich dans ses travaux, en particulier : WAHNICH, Sophie. La pétition : une politisation de la plainte, 1789-1792. Op. Cit. Ce n'est par exemple que dans la Constitution de 1881 que ce droit collectif est reconnu. Même si là encore il est stipulé que les «cinq premiers [signataires] répondront de l'authenticité des signatures et tous de la vérité des faits ». 
pronunciamiento et à la régulation de son usage, tandis que le droit de pétition comme on vient de le voir est encadré, qui plus est de façon restrictive dans la plupart des cas.

Intéressons-nous à présent à la morphologie du pronunciamiento. Ce sont d'abord, comme le laisse entendre l'origine du mot, des déclarations publiques parlesquelles c'est précisément un collectif(et rarement une personne), qui'se prononce', prend fait et cause pour ou contre une situation. Ils ont, à rebours de certaines idées reçues, une signification plus politique que militaire, même si, parmi les instigateurs et les signataires on peut trouver des « hommes en armes » et les différents corps auxquels ils appartiennent. Il est en effet inapproprié de parler, pour une bonne partie du XIXème, de militaires proprement dits, l'armée nationale étant au Venezuela embryonnaire si ce n'est inexistante jusqu'à la fin du siècle et ne se professionnalisant qu'au début du siècle suivant ${ }^{25}$. En témoigne en particulier l'écart saisissant entre le déploiement d'un arsenal législatif et financier important, où tout semble prévu pour que les forces armées soient à même de remplir les missions qui sont les leurs et la réalité du terrain ${ }^{26}$.

Sous sa forme matérielle, le pronunciamiento est la publication formalisée par écrit de l'opinion d'un corps ou d'une corporation plus ou moins formalisée face à une circonstance exceptionnelle. II peut être rédigé dans le cadre du municipe, où des individus, rassemblés par le cabildo, se "prononce" alors pour prendre position, ou lors d'une réunion des membres d'une corporation civile ou armée. Puis, la trame est discutée, circule et elle est réappropriée mais aussi complétée par les différents groupes, corps avant d'être approuvée et sig-

25 IRWIN, Domingo; MICETT, Ingrid. De caudillos a pretorianos. Una Periodización de la realidad militar venezonala, siglos XIX-XX. Nuevo Mundo Mundos Nuevos [En línea], Cuestiones del tiempo presente, Puesto en línea el 31 enero 2011. DOI : 104000/nuevomundo.60783.

26 Comme l'atteste l'analyse des douze volumes de documents sur les forces armées édités par Ramón Velásquez : VELÁSQUEZ, Ramón J. (ed.). Las Fuerzas armadas de Venezuela en el siglo XIX. Caracas : Presidencia de la República, 1971, qui comprennent tous pas moins de 600 pages de textes, discours officiels, règlements et budgets annuels. 
née. Mais il peut également être initié suite à un rassemblement plus spontané de vecinos, de citoyens, même si dans la rédaction du document on retrouve parfois la main d'un notable local.

Il vise à exprimer la position des signataires, d'une communauté face à une situation donnée et à la rendre publique, dans le même temps qu'il rend compte de l'extrême réactivité et mobilisation de ces acteurs sociaux. Quant aux signataires proprement dits, parfois très nombreux, ils comprennent un large spectre d'acteurs : autorités civiles, militaires et ecclésiastiques, vecinos, pères de familles, mais aussi de simples habitants comme en témoignent les nombreuses croix qui font parfois office de signature.

Cette pratique du pronunciamiento, à la différence de la pétition estcontemporainedesindépendances,on peutmêmeconsidérerqu'il s'agit d'une pratique née de la crise impériale. Marie-Danielle Demélas indique d'ailleurs que l'emploi de ce terme pour désigner cette pratique apparaît en 1820 en Espagne, quand le $1^{\text {er }}$ janvier, près de la ville de Cadiz, deux officiers, empruntant le terme au vocabulaire des juristes, l'utilisent pour manifester, par écrit, l'opposition de l'armée menacéed'aller en Amérique pour une guerre, perdue d'avance selon eux. Ils se prononcent contre la politique absolutiste du roi Ferdinand VIII et en faveur de la Constitution de Cadix qu'il a abolie lors de son retour sur le trône en $1814^{27}$.

Elle intervient soit quand les dispositifs propres à la politique légale (notamment le vote et la représentation parlementaire) ont échoué pour se faire entendre ; soit lors d'une crise politique qui suppose, dans un moment de vide de représentation et de recomposition des appartenances à un clan politique, un nécessaire positionnement des différents « corps » vis-à-vis des autorités en place. Le royaume disparu avec la monarchie hispanique, la communauté parfaite que représentait la Monarchie s'était fragmentée en pueblos, et dans certains cas en haciendas. La fréquence des pronunciamientos

27 DEMÉLAS, Marie-Danielle. Retour sur la pratique du pronunciamiento (1820-2013) », Op. Cit., p.43. 
manifeste de cette manière la très grande difficulté de donner un cadre précis à la nouvelle communauté politique. C'est en ce sens que l'on peut considérer cette « pratique comme une réponse à la rupture des pactes traditionnels de réciprocité qui règlent les relations entre l'État et les pouvoirs locaux ».

Cette véritable prise de parole collective et publique, qui fait opinion et fait effraction dans une sphère publique où la parole, populaire tout particulièrement, est extrêmement contrôlée ${ }^{28}$, est complémentaire et non contradictoire avec les formes légales et constitutionnelles d'expression de la « souveraineté ». En outre, dans la mesure où elle va bien au-delà du corps électoral stricto sensu, elle nous informe sur les logiques de mobilisation de ces acteurs en conflit qui sont encore, pour l'essentiel, des acteurs traditionnels, héritiers de la société d'ancien régime et des luttes pour l'indépendance, mais exclus pour la plupart du corps politique. Le pronunciamiento signifie bien en ce sens la simulation d'une dissolution du corps politique et de sa refondation par le biais de pactes librement consentis par les communautés de base, témoignant ainsi de l'agentivité d'une large frange de la population non citoyenne.

Comprenant une déclaration préliminaire, qui constitue souvent la matrice du pronunciamiento, celle-ci est reprise dans les documents rédigés par les différents corps qui expriment en y insérant leur propre lecture des faits et des décisions propres à leur communauté. Très souvent, ces pronunciamientos sont écrits sur du papier timbré comme n'importe quel document officiel et parfois publiés dans la presse, voire réunis et édités sous forme de livre comme c'est le cas de l'une des campagnes étudiées dans cet article.

On est donc loin de l'image souvent véhiculée d'une conspiration de quelques conjurés appuyés par la troupe. L'acte qui réunissait le

28 Voir HÉBRARD, Véronique. Opinion publique et représentation dans le Congrès Constituant vénézuélien (1810-1812). Annales Historiques de la Révolution Française, n. 365 « Lumières et révolutions en Amérique latine », Juillet-septembre 2011, p.153-175. Pour une approche plus globale de cette question on peut consulter FARGE, Arlette. Essai pour une histoire des voix au dix-huitième siècle. Paris : Bayard éditions, 2009. 
peuple de chaque lieu de la république pour décider de la rupture et de la rénovation du pacte n'avait rien de clandestin. II reproduisait l'imaginaire qui structurait l'espace ancien : une fédération de pactes entre communautés territorialisées, s'inscrivait dans un réseau de sociabilité et de fidélités anciennes qui n'avaient pas besoin du secret.

Ces campagnes fonctionnaient à la manière de vastes consultations des populations, mettant en évidence la permanence de la culture « pactiste » de refondation, mais non codifiées, c'est en ce sens qu'elles participent, de mon point de vue, de ces « autres lieux du politique » dont la légalité est nulle, mais dans la légitimité et la performativité est indéniable.

D’où ce décalage entre l'absence de légalité du pronunciamiento et son rôle de régulation ; sa capacité d'introduire du « désordre » dans la vie politique et sa reconnaissance voire son usage y compris par les autorités à des fins de légitimation.

On peut donc considérer que l'art du pronunciamiento vénézuélien concerne pour l'essentiel une prise de position collective de larges secteurs de la population, masculine pour l'essentiel, face à une situation politique exceptionnelle. C'est pour le moins le cas des deuxcampagnes de pronunciamientos repérées dans le cadre de cette étude.

\section{Exprimer des Votos et pronunciarse dans le Venezuela de 1828}

Lapremière de ces campagnes estlevastemouvement desoutien qui se développe en faveur de Bolivar en 1828, dans les départements Vénézuéliens de la République de Colombie, lorsque la menace de dissolution est à son comble. D'où un appel à l'homme providentiel (Simón Bolívar) et la volonté exprimée par écrit, qu'il exerce des pouvoirs dictatoriaux au sens ancien du terme. En effet, cette campagne se produit à l'occasion de la réunion de la Grande Convention destinée à œuvrer au maintien de l'intégrité territoriale de la République, mais aussi de son retour à Caracas. Les cités et les corps émettent alors leurs Votos en réponse à l'appel lancé par Páez à l'approche de la réunion de cette Grande Convention. 
Outre les débats officiels et dans la presse, cette parole s'exprime en premier lieu dans plus d'une centaine de actas et representaciones rédigés à l'initiative de corps municipaux, militaires, de « pères de famille », de citoyens ou de vecinos, retranscrits pour certains dans la presse, mais surtout pour leur grande majorité, regroupés dès 1828 dans le volume 13 de la Colección de documentos relativos a la vida pública del Libertador de Colombia y del Perú Simón Bolívar ${ }^{29}$ puis, dans un petit ouvrage et par le même éditeur en 1829, intitulé de façon très symptomatique: El Voto de Venezuela. O colección de actas y representaciones de las corporaciones civiles, militares y padres de familias de los departamentos de Venezuela, Maturin y Orenoco, dirigida a la Gran Convención de Colombia y a SE el Libertador Presidente sobre reformas ${ }^{30}$. Ce volume comprend au total 100 Representaciones et Actas, 45 émanant de collectifs de civils, en premier lieu des Municipalités et quelques paroisses, des vecinos, padres de familia, notables, mais aussi des représentants de l'Université de Caracas, des administrateurs des douanes. Les 55 autres sont rédigés par les différents corps armés, bataillons de milices, commandants et officiers de différentes armes (Artillerie, Cavalerie, Marine), Escadrons civiques. Dans un seul cas, la Representación est mixte ${ }^{31}$.

L'intérêt de cette campagne, outre sa double publication quasi simultanée et donc sa médiatisation, est qu'elle constitue une longue série, permettant ainsi de modéliser les observations à partir de la répétition de certains effets de langage, d'un champ lexical particulier,

29 Coleccion de documentos relativos a la vida publica del Libertador de Colombia y del Perú Simón Bolívar. Para servir a la historia de independencia del Suramérica. Caracas: Imprenta de G. F. Devisme, 182818, Tomo decimotercio, 373p.

30 El Voto de Venezuela. O colección de actas y representaciones de las corporaciones civiles, militares y padres de familias de los departamentos de Venezuela, Maturin y Orenoco, dirigida a la Gran Convención de Colombia y a SE el Libertador Presidente sobre reformas. Caracas: Devisme, $1829,315 \mathrm{p}$. Sauf mention contraire tous les Actas et Representaciones sont extraits de ce volume.

31 Representación de los Gefes, oficiales, padres de familias y Vecinos propietarios del Cantón de Aragua, 16 de abril de 1818. In: Coleccion de documentos relativos a la vida publica del Libertador de Colombia y del Perú Simón Bolivar. Para servir a la historia de independencia del Suramérica. Op. Cit., p.315-317. Notons que cette Représentation ne figure pas dans le volume publié en 1829, ainsi que quelques Actas de municipalités colombiennes et celui de la ville de Quito. 
de références communes, mais aussi du récit des modes d'action et réactions qui ont entouré la rédaction et l'approbation des différents textes.

Concernant tout d'abord cet appelà Bolivarquel'on peut liredans la majoritédes documents, ses modalités d'énonciation attestent qu'il renvoie à cette nécessaire incarnation du pouvoir, présentée comme la meilleure garantie du rétablissement de l'unité et comme l'unique rempart contre "l'anarchie ", terme qui est très fréquemment employé, mais aussi contre les irrégularités qui auraient prévalu pour l'élection des députés à la Convention. En vertu de son prestige militaire et de la véritable vénération dont il est l'objet depuis qu'il a été fait Libertador, Bolívar apparaît en effet comme le seul à même de rétablir l'ordre et la sécurité, d'éviter le retour du fédéralisme que souhaiterait, selon ces écrits, rétablir Santander et ses partisans. D'où ce recours au chef tutélaire unique, autoritaire et, dans le même temps, pacificateur ; caractère impérieux d'un pouvoir personnel et concret, à l'image du monarque.

Au-delà des éléments de contexte, l'analyse approfondie de ces écrits nous informe donc sur la culture politique de ces collectifs, civils, militaires, corporatifs, mais aussi ensemble de vecinos et padres de familia, leur lecture de ce contexte, les références mobilisées mais aussi leur conception du Souverain. Un homme qui doit être charismatique et dont l'obligation première est d'être physiquement près de ceux qu'il dirige. Surtout, au hasard des formulations ce sont les mécanismes mobilisés pour se réunir, rédiger ces textes ou les reformuler, se faire entendre des corps constitués (le cabildo en premier lieu) mais aussi légitimer cette prise de parole qui nous intéressent.

Arrêtons-nous à présent sur la façon dont ces différents acteurs, collectifs et individuels, expriment leurs critiques à l'égard des conditions dans lesquelles les membres de la Convention ont été élus, mais surtout leur souhait en façon de Bolívar comme seule solution à mêmede sauverla République et la mémoire de la lutte menéedepuis 1810 pour conquérir l'indépendance de l'Espagne. 
On retrouve dans la plupart des Votos et Representaciones une aspiration profonde, exprimée en termes où l'émotionnel le dispute aux arguments factuels, au pouvoir « paternel, d'ordre et de moral, unique rempart pour les vertus, le succès et la splendeur de la Patrie » comme le mentionne la municipalité de Barquisimeto ${ }^{32}$. On lui demande « autorité » et " protection », en vertu des qualités qui lui sont conférées. Il est considéré comme étant le seul à pouvoir mettre fin au « chaos », à « l'anarchie » dans lesquels la Colombie est en train de plonger. Les menaces extérieures, les dissensions internes, l'abus de confiance face au manque supposé de gens suffisamment éclairés pour faire adopter le fédéralisme : si l'on ne prend pas de mesures énergiques pour mettre fin aux dangers qui menacent la nation, c'est le futur même de la Révolution qui se trouvera compromis. Ce sont surtout les Représentations de chefs de bataillons de milices qui insistent sur ce dernier point, car ils ne veulent pas s'être battus pour rien :

«Si dans ces circonstances le pouvoir est divisé, les moyens de défense deviendront inefficaces, et la Colombie tombera tout d'abord dans l'anarchie, puis sous la domination de ses anciens métropolitains. Le sang de tant de Colombiens aura coulé inutilement durant la guerre d'indépendance ; et les titres glorieux acquis par la République grâce à elle, seront l'objet de la moquerie et du mépris. Il est donc nécessaire, pour que la grande œuvre de 18 ans soit conservée et ne tombe pas en dérision, de la soutenir avec un gouvernement de vigueur et d'unité. ${ }^{33}$

Avec la référence à l'œuvre révolutionnaire, dont les partisans et acteurs ne peuvent admettre qu'elle soit réduite à néant du fait de l'anarchie et des dissensions civiles qui agitent certaines parties du territoire, s'articulent les deux facettes du personnage de Bolívar : le

32 Acta de la Municipalidad de Barquisimeto, 13 de marzo de 1828. El Voto de Venezuela. Op. Cit., p.131. 33 Representación de los jefes y oficiales del batallón de milicias auxiliares $n^{\circ} 1$, a S.E. el Jefe Superior de Venezuela, marzo de 1828. Op. Cit., p. 10-11. Voir aussi Representación de los jefes y oficiales del batallón de milicias arregladas $n^{\circ} 12$ a S.E. el Jefe Superior de Venezuela. Los Teques: 3 de marzo de 1828. Op. Cit., p.27-28. 
chef armé et l'homme politique, dans sa fonction de président de la République de Colombie. Deux facettes antithétiques.

Au-delà du culte qui lui est rendu à cette occasion, ce qu'il me sembleimportant demettreen évidence c'est le lien établi entre prouesses militaires et les capacités politiques qu'on lui prête, puisqu'il est celui qui par « son épée et ses talents nous a élevés au rang de nation » 34. De la même façon, la Municipalité de Píritu déclare:

«Bolívar saura, l'épée dans une main et le code dans l'autre, redonner aux fils de Colón I'honneur qu'ils ont perdu du fait des agitations continuelles $»^{35}$.

Du fait de son rôle dans la guerre, il est considéré comme le « créateur de la nation $»^{36}$, de la « République ${ }^{37}$, celui qui, « en tant que père créateur et fondateur » de la Colombie « a le souhait le plus vif de la sauver des risques qui la menacent ${ }^{38}$. II est même érigé par certains comme le « sauveur d'une partie considérable du genre humain ${ }^{39}$, même si, pour d'autres il est encore plus que le créateur de la nation. Comme l'affirment certains officiers « pour nous la personne de Bolívar et la Patrie c'est la même chose ; (...) l'une sans l'autre ne peut exister ${ }^{40}$. Une telle identification et dépendance de la nation, mais aussi de la République et de la patrie avec la personne de Bolívar

34 Acta de los Comandantes y Oficiales del batallón auxiliar n. ${ }^{4}$ de Barlovento, a S.E. el Jefe Superior de Venezuela. Caucagua: 22 de marzo de 1828. Op. Cit., p.214-215.

35 Representación de la Municipalidad de Píritu, 22 de abril de 1828. Op. Cit., p.307.

36 Representación de le padres de familias y propietarios de Caracas. Op. Cit., p.24.

37 Representación de los notables y padres de familias de Petare dirigida a la muy ilustre Municipalidad. Op. Cit., p.44 ; Representación de los Gefes y oficiales del Batallón y Milicias auxiliar de Cumaná. Op. Cit., p.54.

38 Acta de la Municipalidad de Santa Cruz de Caucagua. Op. Cit., p.218.

39 Representación de los Jefes y Oficiales del batallón de milicias arregladas del cantón de Quibor a S.E. el presidente de Colombia, marzo de 1828. Op. Cit., p.91.

40 Representación de los Jefes Oficiales del regimiento de los lanceros de La Victoria, provincia de Apure, a S. E. Libertador presidente. Achaguas: 16 de marzo de 1828. Op. Cit., p.197. 
ne pouvaient que rendre plus patent le discrédit du gouvernement et de la Convention. Il apparaît comme l'incarnation du Souverain. En ce sens, la déclaration de l'Escadron civique de Caracas n'est que plus significative.

« Nous ne trouvons d'autre lumière qui nous guide, ni d'autre main qui nous arrache de la tombe, que (...) le génie sublime de SE le Libertador Presidente. C'est lui qui a créé la Colombie, il doit donc la sauver de I'anarchie, la régénérer et lui donner des lois, des coutumes, la dignité, la splendeur, la liberté et de nouvelles gloires ${ }^{41}$.

Mais, au-delà du culte lui-même, recourir de la sorte à Bolívar témoigne du caractère ténu de l'ancrage de la représentation moderne revendiquée par ailleurs. A travers une telle foi dans le pouvoir personnel d'un seul, outre l'homme en arme, le sauveur de la patrie, doté des qualités quasi démiurgiques d'un monarque, se manifeste une culture politique attachée à l'incarnation de la souveraineté ${ }^{42}$.

Outre leur contenu qui nous renseigne donc sur la lecture d'un contexte, les solutions proposées et par conséquent sur leur représentation et culture politique, ces documents permettent également de retracer leur genèse ainsi que les formes d'interactions et de sociabilité qui en ont accompagné la rédaction.

Arrêtons-nous en premier lieu sur la façon dont ces différents acteurs justifient avoir pris la plume.

Alors que, comme signalé plus haut, aucune référence explicite au droit de pétition ne figure dans les constitutions de 1819 et 1821,

41 Representación del escuadrón cívico de Caracas al Jefe Superior de Venezuela. Caracas: 4 de marzo de 1828. Op. Cit., p.30-31.

42 Comme en témoigne le travail réalisé par Ariel de la Fuente sur les chansonniers du Chaco argentin et qui permet de saisir, par le bas, l'incorporation de cette pratique par les acteurs, ainsi que cette tension entre représentation moderne et souveraineté incarnée. DE LA FUENTE, Ariel. Facundo and Chaco in Songs and Stories: Oral Culture and the Representations of Caudillos in the Nineteenth-Century Argentine Interior. Hispanic American Historical Review, 80:3, 2000, p.503-535. 
ce droit est à plusieurs reprises invoqué dans les textes pour justifier cette prise de parole et sa consignation par écrit afin qu'elle soit connue des autorités locales, supérieures mais aussi et tout particulièrement de Simón Bolívar. C'est le cas plus d'une dizaine de fois, aussi dans des Représentations ou Actas de corporations civiles que de corps armés.

Ainsi, tandis que le $1^{\text {er }}$ mars les signataires de la Représentation du Bataillon Callao font valoir le « droit de pétition dont disposent tous les Colombiens en vertu de la même constitution ${ }^{43}$, les chefs et officiers du Bataillon de Milices de los Teques s'expriment en référence explicite à la Constitution de Cucutá, dont ils « auraient privé l'acte inconstitutionnel réalisé pour le décret de convocation expédié pour réunion la grande convention ${ }^{44}$. II en va de même des Padres de familia y propietarios de Caracas qui, face à la situation critique du pays, se prévalent eux aussi « du droit de pétition » dont «ils s'empressent de se prévaloir pour exprimer en toute liberté (...) nos opinions au Père de la Patrie ${ }^{45}$. Plusieurs autres évoquent uniquement le droit de pétition en tant que tel, sans référence explicite à ce qui existerait dans la constitution, à l'instar des Chefs et Officiers de marine de Puerto Cabello ${ }^{46}$ ou de ceux de l'escadron des dragons de l'Alto Llano ${ }^{47}$, mais aussi des vecinos d'Ocumare ${ }^{48}$. Les Commandants et Officiers du Ba-

43 Representación del Batallón Callao a S. E. El Libertador Presidente de Colombia. Caracas: 1 de marzo de 1828. El Voto de Venezuela. Op. Cit., p.1.

44 Representación de los Gefes y oficiales del Batallón de Milicias arregladas n 12, Los Teques, 3 de marzo de 1828. Op. Cit., p.27.

45 Representación de los padres de familias y propietarios de Caracas. Caracas: 2 de marzo de 1828. Op. Cit., p.22.

46 Representación de los Gefes y oficiales de Marina de Puerto Cabello, Puerto Cabello: 15 de marzo de 1858, Op. Cit., p.171.

47 Representación de los Gefes y oficiales del Escuadrón Dragones del Alto Llano. San Rafael de Orituco: 18 de marzo de 1828. Op. Cit., p.207.

48 Representación de los vecinos del Cantón de la Costa de Ocumare. Ocumare de la Costa: 21 de marzo de 1828. Op. Cit., p.213. 
taillon auxiliaire de Barlovento parlent même « du droit sacré de pétition ${ }^{49}$.

Ils sont en revanche moins nombreux à employer le terme « pronunciamiento » pour qualifier la nature de leur représentation. Nous le trouvons notamment dans la très longue Représentation des Chefs et Officiers de la Garnison de Puerto Cabello ${ }^{50}$ mais aussi dans celle de la municipalité de San Fernando de Apure dont les membres réunis en Cabildo extraordinario parlent d'un « pronunciamiento en faveur du père de la Colombie ${ }^{51}$. Certains le font avec ferveur comme tiennent à le mentionner les auteurs de la Représentation de l'escadron civique de Caracas qui concluent leur texte en affirmant que « tel est le pronunciamiento clair, exprès, spontané et décisif » ainsi que celui « de leurs fils $»^{52}$. A S. Rafael de Orituco, ce sont les chefs et officiers d'un bataillon de cavaleriequi, dans leur Représentation, «demandent que I'on accueille avec bienveillance ce pronunciamiento libre et déterminé $»^{53}$. Pour sa part, la ville de Guarenas se « prononce » lors de la réunion du cabildo et de « la plus grande partie du vecindario, le cœur de chacun des participants enflammé du noble orgueil de faire partie de ceux dont la postérité se souviendra $»^{54}$. Enfin, dans la petite ville de Piritu, chef du canton du même nom, dans la province de Barcelona, c'est le Síndico Procurador qui s'exprime au nom du collectif et fait part de « sa satisfaction d'avoir émis un pronunciamiento, fils de la bonne

49 Representación de los Comandantes y oficiales del Batallón Auxiliar $n^{\circ} 4$ de Barlovento. Caucagua:

22 de marzo de 1828. Op. Cit., p.214.

50 Representación de los Gefes y oficiales de la Guarnición de Puerto Cabello. Puerto Cabello: 8 de marzo de 1858. Op. Cit., p.71.

51 Representación de la Municipalidad de San Fernando de Apure. San Fernando de Apure: 17 de marzo de 1828. Op. Cit., p.228.

52 Representación del Escuadrón Cívico de Caracas. Caracas: 4 de marzo de 1828. Op. Cit., p.31.

53 Representación del Gefe y oficiales del escuadrón n 1. Granaderos a Caballo de la Guaira. S.

Rafael de Orituco: 9 de marzo de 1828. Op. Cit., p.89.

54 Acta de la Municipalidad de Guarenas. Guarenas: 16 de marzo de 1828. Op. Cit., p.203. 
intention qui me caractérise, pour le salut de la patrie $»^{55}$. L'Acte se conclut en des termes des plus éloquents:

"La municipalité, après une discussion approfondie, et guidée l'impulsion de son enthousiasme et patriotisme, mit l'exposition du Sindico dans la balance de la justice et se mit d'accord sur le fait qu'elle I'approuvait totalement pour correspondre aux votos que les vecinos ont émis spontanément. $\rangle^{56}$

Unefois ces positionnements effectués, c'est le déroulé de la prise de parole et de sa transcription qu'il nous est donné à lire mais aussi à entendre, sentir, palper tant ces documents sont vivants, tout particulièrement les Actas et Representaciones des Municipalités qui relaient très souvent les propres actes de leurs concitoyens qui participent à leurs réunions dans la plupart des cas et dont ils sont fréquemment à l'origine.

Certaines municipalités revêtent ce moment d'une certaine solennité en convoquant un Cabildo Extraordinario. Décision prise après avoir été alertée par la réception « d'une pétition de plusieurs vecinos» ayant manifesté leur inquiétude face à la situation de la République. C'est notamment le cas de la municipalité de Petare qui, réunie le 7 mars 1828 devant les vecinos notables et les auteurs de la pétition, décide après une nouvelle lecture du texte mais aussi discussion de son contenu, décide de la publier en son nom ${ }^{57}$. Deux jours plus tard, elle

55 Acta de la Municipalidad del Cantón de Piritu. Villa de Piritu: 22 de abril de 1828. Op. Cit., p.308. 56 Ibidem, p.308-309.

57 Acta de la Municipalidad de Petare. Villa de Petare: 7 de marzo de 1828. Op. Cit., p.46-47. 
rend compte d'une nouvelle réunion avec d'autres vecinos du canton, afin là encore de discuter de leur « représentation ». Il est là encore procédé à une lecture du texte, dont « le contenu fit sentir à tout l'auditoire, l'urgente nécessité de trouver par tous les moyens licitement permis, à s'opposer à d'aussi grands désastres ». S'ensuit, avant la résolution finale, la prise de parole « avec beaucoup d'enthousiasme, de certains des présents » et la condamnation des « sinistres machinations des anarchistes, créatures de l'abominables administration ${ }^{58}$.

La municipalité de Yaritagua se réunit pour sa part le 12 mars à la demande de citoyens qui, le matin même, se sont présentés à cette fin chez le Secretario. Elle fait également le choix de convoquer un Cabildo extraordinario en la présence de ces « nombreux citoyens parmi les plus respectables du canton », afin qu'ils viennent exprimer les raisons de leur mobilisation ${ }^{59}$. Les membres du cabildo, après les avoir entendus, indique ne pas « pouvoir être indifférents aux clameurs du pueblo qu'il représente » et décide «d'adresser l'original de leur représentation à l'Excmo Sr. Libertador Presidente ${ }^{60}$. II est ensuite mentionné que l'Acte s'est conclu « dans le calme», " au milieu des tendres démonstrations de joie » des présents qui apposent leur signature en bas du document qui, rajoutant à la solennité de l'écrit, a été rédigé sur « du papier de cinquième sceau, ne disposant pas du sixième ${ }^{61}$.

A Quivor, où la municipalité se réunit également en Cabildo extraordinario, compte parmi les présents des « pères de famille et notables de la bourgade ${ }^{62}$. Dans I'Acte final adressé à SE el Libertador Presidente, figurent de nombreux détails qui nous renseignent sur les pratiques mises en œuvre préalablement à sa rédaction. Il est en effet précisé qu'il y eut tout d'abord une « longue discussion puis un examen des différentes opinions émises, oralement comme par écrit »

58 Acta de la misma y Vecinos del Cantón. Villa de Petare: 9 de marzo de 1828. Op. Cit., p.48.

59 Acta de la Municipalidad de Yaritagua. Villa de Yaritagua: 12 de marzo de 1828. Op. Cit., p.114. 60 Ibidem, p.115-116.

61 Ibidem, p.116.

62 Acta de la Municipalidad de la Villa de Quivor.Villa de Quivor: 14 de marzo de 1828. Op. Cit., p.174. 
mais aussi « dans plusieurs journaux et d'autres imprimés, sur la forme du gouvernement et la réforme de la constitution qui doit être adoptée lors de la réunion de la Grande Convention $»^{63}$. Réforme dont ils jugent ne rien pour voir attendre.

Certaines de ces réunions se terminent par des applaudissements, dans et en dehors de l'enceinte de l'ayuntamiento, comme à Santa Cruz de Caucagua dont la municipalité se réunit en cabildo ordinario le 22 mars 1828 :

« Unanimes, les membres de cette corporation décidèrent faire part des votos exprimés à l'intention de la Grande Convention, en adressant la minute de ce procès-verbal à l'intendant départemental (...), ce qui fut applaudi avec de vives acclamations en faveur du Libertador Presidente par une grande partie du pueblo qui était en train d'écouter la session (...). ॥ $^{64}$

Les propos des vecinos de Puerto Cabello nous renseignent de façon originale et affirmée sur le sens de cette prise de parole collective. En effet, au moment de la réunion des membres de leur municipalité ils tiennent à protester pour ne pas avoir pu se réunir préalablement afin de formuler leur représentation ${ }^{65}$. Dénonçant plus généralement la violation dela loi par legouvernement de Bogotá, ils adressent donc cette requêteà municipalité; requête qui plus est signée par plusieurs membres de la milice qui « malade au moment de signer la militaire signe [cette représentation] comme citoyen », en témoignage de leur soutien ${ }^{66}$. Enfin, et c'est assez exceptionnel, après la liste des signataires, dans un Addenda la municipalité mentionne les faits suivants,

63 Ibidem.

64 Acta de la Municipalidad de Santa Cruz de Caucagua. Santa Cruz de Caucagua: 22 de marzo de

1828. Op. Cit., p.218.

65 M. I. Municipalidad. Puerto Cabello: 13 de marzo de 1828. Op. Cit., p.144.

66 Ibidem, p.151. 
permettant de façon très intéressante de nous restituer son ambiance visuelle et sonore :

«Parmi la présence de nombreuses personnes parmi les plus notables du pueblo, plusieurs prirent la parole alternativement, et ratifièrent avec des discours énergiques le contenu de la représentation et affirmèrent ici et devant le monde entier que leurs votos étaient irrévocables; et l'un d'eux ayant demandé que l'on proclame le Libertador comme l'unique et suprême représentant provisoire du peuple, l'idée fut applaudie et tous poussèrent de cris de joie et des vivats $(. ..) »^{67}$.

Enfin, prenant acte de ce qui a été énoncé et après avoir décidé d'insérer cette pétition, la façon dont les membres de la municipalité formulent leur conclusion renvoie bien à cette nécessaire rupture du pacte, à cette nécessité de « renoncer ou suspendre pour le temps nécessaire la forme de gouvernement qui nous a régis jusqu'à présent » afin de le refonder sur de nouvelles bases ${ }^{68}$.

Nombreux sont ainsi les indices nichés dans ces documents qui témoignent des échanges entre différents secteurs et acteurs de ces villes et villages qui se réunissent à leur demande très souvent au sein des cabildos, pour expliquer les raisons pour lesquelles ils ont décidé de rédiger un texte, une pétition. Ils se retrouvent donc certes pour apposer leur signature en bas de l'Acte ou de la représentation rédigé à son tour par le corps municipal, mais après avoir débattu, partagé des opinions, parfois avec ferveur ou émotion, s'être informé en lisant les journaux, preuve de véritables formes de sociabilités politiques locales. Il en va de même au sein des corps armés de tous types dont les textes donnent lieu à débat, sont adressés parfois à la Municipalité qui les mentionne, voire les insère, dans son propre écrit.

67 Ibidem, p.152.

68 M. I. Municipalidad. Puerto Cabello: 13 de marzo de 1828. Op. Cit., p.153. 


\section{8 : se prononcer pour la destitution d'un président}

La seconde campagne, beaucoup moins médiatisée que la première ${ }^{69}$, peut-être parce que la Guerre Fédérale éclate quelques mois après, concerne les pronunciamientos rédigés suite à la destitution du président de la République, José Tadeo Monagas en mars 1858, pour en célébrer la chute et dénoncer les excès commis durant son « règne ». De fait, j'ai localisé de nombreux pronunciamientos rédigés et signés au cours des mois de mars et d'avril 1858 afin, pour les rédacteurs et signataires, d'une part d'apporter leur « soutien » à l'arrivée de Castro au pouvoir ; d'autre part d'exprimer leur rejet des Monagas et de leurs pratiques. Au même moment, presque seuls contre tous, trois hommes constituent une faction armée, la Faction de la Sierra, initialement pour apporter leur soutien au président déchu. Cette seconde série, du milieu du siècle, je l'ai découverte à travers mes travaux sur une faction armée, la Faction de la Sierra dont la constitution est contemporaine de la chute, en mars 1858, du président José Tadeo Monagas ; Faction dont les chefs sont de fervents partisans ${ }^{70}$.

Plus artisanaux dans leur matérialité, même si quelques-uns ont été publiés dans la presse ${ }^{71}$, ils fournissent des informations intéressantes sur ce moment de crise, mais aussi et là encore des données précieuses sur les modalités de leur rédaction, les conditions de recueil des signatures, mais aussi sur les formes de sociabilité qui se déploient au long des différentes étapes de leur élaboration. De plus, la longue traque dont est l'objet la Faction, entre mars 1858 et octobre 1859, nous ramèneà ces pronunciamientos età ceuxquilesont signés.

69 Ces pronunciamientos se trouvent pour l'essentiel à l'Archivo General de la Nación/Caracas-Interior y Justicia, tomos DCXVI et DCXIX.

70 Voir HÉBRARD, Véronique. Dinámicas de movilización y lógicas de socialización de lo político en el mundo rural venezolano, 1858-1859. Illes $i$ imperis, n. 17, 2015, p.113-136.

71 C'est notamment le cas de celui de Mérida : Pronunciamiento de la Ciudad de Mérida. Mérida: Imprenta de la Gran Convención Nacional por Juan de Dios Picón Grillet, 25 de marzo de 1858. 
Ces pronunciamientos énoncent en premier lieu une prise de position, une forme de résistance à la façon dont les Monagas ont envisagé l'exercice du pouvoir, en violation selon les auteurs de ces pronunciamientos, des principes républicains. Ce qui marque un changement significatif en termes de formes d'énonciation du politique par rapport à la campagne de 1828. Les pronunciamientos de 1858 ont en effet ceci de caractéristique que même s'ils dénoncent les années Monagas et les failles du régime, tout en exprimant leur soutien à Julián Castro (à sa volonté de marcher sur Caracas, puis de prendre le pouvoir), on n'y retrouve pas, en revanche, cet appel à l'homme providentiel qui, par son seul charisme, pourrait sauver le pays, comme nous avions pu le noter en 1828. À l'inverse ici, les modes d'énonciation permettent de saisir la façon dont ces acteurs se sont en quelque sorte approprié certaines figures théoriques de la modernité politique et les formes d'intelligibilité du politique qui sont les leurs.

Ces pronunciamientos témoignent non seulement de la réactivité aux événements en cours, mais aussi des modalités d'énonciation de leur positionnement politique dans ce contexte de crise politique aigue. Ils nous renseignent sur la façon dont les communautés réagissent à la crise qui se joue durant ce mois de mars 1858 et aux conséquences du départ du président JoséTadeo Monagas le 15.De même, ils fourmillent d'informations sur les modalités mises en œuvre pour la réunion des différents acteurs, puis pour la rédaction, la discussion et l'approbation du texte des différents pronunciamientos.

Dans plusieurs d'entre eux, par exemple, les modalités et les délais pour venir apposer sa signature sont précisés de façon détaillée, ainsi que le lieu où le document est à disposition. Les auteurs de celui de la Paroisse de Zaraza, dans la province du Guárico, mentionne ainsi « que cet acte sera ouvert pour trois jours avec l'objectif que puissent venir le signer tous les individus qui, n'ayant pas pu participer à la session de ce jour, souhaiteraient le faire ${ }^{72}$. Les vecinos de

72 Pronunciamiento de la « Parroquia de Zaraza, cabezera del Canton Unare, provincia del Guárico, a los 23 dias del mes de Marzo de 1858 », AGN/Caracas-Interior y Justicia. Tomo DCXVI, fol. 271. 
Chaguaramas qui rédigent leur pronunciamiento le laissent pour leur part 48 heures à ceux qui souhaiteraient venir apposer leur signature, même si des copies de ce pronunciamiento seront immédiatement faites pour être diffusées aux autorités et les faire circuler dans les villages avoisinant ${ }^{73}$.

Tout est fait effectivement pour qu'une large publicité soit faite à ces prises de parole dans le même temps qu'on en vient à leur conférer une fonction d'autorité et une certaine probité à certains de leurs signataires. En juillet 1858, on apprend, lors de l'interrogatoire d'un homme suspecté d'appartenir à la Faction, qu'un pronunciamiento a été rédigé dans «le village de San José » de Tiznados dont est originaire et où réside l'accusé ${ }^{4}$. En effet, il s'y réfère pour dire que le fait de l'avoir signé, est une preuve de son innocence. Cette fonction de disculpation joue également pour le Colonel Lapalma dont le Chefd'Etat major en charge des opérations dans les Llanos prend la défense en ces termes:

«(...) le Sr Colonel Lapalma n'a jamais été uni aux factieux, bien au contraire, il fut celui qui se mit à la tête du pronunciamiento de San Fernando [d'Apure], se reconnaissant dans le mouvement général de la Nation. $»^{75}$

D'ailleurs, le Commandant du Canton de El Pao chargé de transmettre cette information au gouverneur de la province tient lui aussi à apporter son soutien au Colonel en des termes encore plus affirmés et rudes envers le président déchu, puisqu'il déclare que le Colonel

73 Pronunciamiento de la «Villa de Chagaramas a diez y seis de Marzo de 1858 », AGN/Caracas-Interior y Justicia. Tomo DCXVI, fol. 274.

74 Interrogatoire de Pablo Acosta. Estado Mayor de operaciones de Guarico y Apure, San Francisco de Tisnados, 27 juillet 1858. AGN/Caracas-Interior y Justicia. Tomo DCXVII, fol. 413v.

$75 \mathrm{Le}$ Sr Jefe de E. Mayor de la Division de operaciones de los Llanos, informe le Comandante de Armas del Canton Pao (Julian Ramos), qui informe le Gouverneur de la province de Valencia (Carlos P. Calvo, hijo), Valencia, 5 de abril de 1858. AGN/Caracas-Interior y Justicia. Tomo DCXVII, fol. 294v. 
Lapalma, « loin de défendre la cause du Tyran, comme on l'a affirmé, a dignement partagé le pronunciamiento des pueblos, se mettant à la tête de celui de San Fernando de Apure ${ }^{76}$.

Parmi les quinze pronunciamientos sur lesquels j'ai travaillé, cinq sont antérieurs à la chute de Monagas, un est daté du jour même de son renoncement au pouvoir, et les neuf autres s'échelonnent du 16 au 30 mars. Ils sont l'œuvre, dans la plupart des cas, des corps municipaux, mais aussi de vecinos ou de représentants de corps armés. Tous appellent à la signature de leur texte par le plus grand nombre de personnes, laissant à cette fin les registres ouverts pendant deux ou trois jours selon les cas. Les pronunciamientos émanant de localités situées dans la province d'Aragua sont ainsi transmis à Caracas par le gouverneur le 8 avril, en des termes ratifiant, s'il en était besoin, que ces pratiques et ces écrits participent pleinement de la vie politique, même s'ils n'ont aucune légalité.

"Je vous joins les pronunciamientos qui se sont tenus dans les villes de Maracai, Cura et Turmero, et dans les paroisses de Choroni, Magdalena et San Juan de los Morros, désavouant le gouvernement calamiteux du Général JoséTadeo Monagas, reconnaissant le Gouvernement provisoire et demandant une Convention Nationale qui reconstitue la République sous des formes véritablement républicaines, se conformant ainsi au reste de la Nation vénézuélienne (...). $»^{77}$

De fait, ces pronunciamientos, de façon plus appuyée dans les 5 premiers, sont d'une grande virulence à l'encontre de Monagas le "Tyran » et de sa "dynastie », dénonçant les violations de la loi et de la constitution, le manque de liberté d'expression et de presse, la corruption de la justice, les usurpations de terres, la dilapidation et

76 Le Comandante de Armas del Canton Pao (Julian Ramos). Al Gobierno Superior Político de la Provincia de Carabobo (Carlos P. Calvo, hijo). Al Secretario de E en los DD del Interior y Justicia, Valencia, 5 de abril de 1858. AGN/Caracas-Interior y Justicia. Tomo DCXVII, fol. 295.

77 Gobernador de la Provincia d'Aragua. Al Señor Sec de E. en los Despachos del Interior y Justicia, La Victoria, 8 de abril de 1858. AGN/Caracas-Interior y Justicia. Tomo DCXIX, fol. 287. 
l'usurpation du Trésor Public, les violences, les assassinats et les humiliations. Ce qui amène les vecinos de la paroisse de Parapara à se dire « persuadés que la domination des Monagas est contraire aux principes républicains (...) et ne dispose pas de l'appui de la Volonté Nationale ${ }^{78}$, et que l'administration de José Tadeo est, pour ceux de Camaguán « attentatoire à nos institutions républicaines depuis l'abominable 24 janvier 1848, où il a assassiné les représentants de la Nation désarmés (...) ॥ ${ }^{79}$. Mais, au-delà du présent, c'est aussi la trahison des valeurs de l'indépendance qui est dénoncée:

"Les Monagas se sont arrogé le droit exclusif de gouverner le peuple du Venezuela, ce même peuple qui a époustouflé le monde avec les faits les plus héroïques lors de la glorieuse guerre de l'indépendance, le peuple de Bolívar et de tant d'hommes illustres qui, avec une abnégation extraordinaire, ont préféré la mort à l'esclavage, et qui sont enfin parvenus, après mille combats, à détruire les chaînes du despotisme, à vaincre les vainqueurs du Grand Capitaine qui a asservi l'Europe et à ériger des temples à l'Egalité, à la Liberté et à la Fraternité, temples scandaleusement profanés par ceux qui aujourd'hui s'appellent nos seigneurs, nos maîtres (...). . ${ }^{80}$

Les pronunciamientos postérieurs au 15 mars dénoncent eux aussi les errements des Monagas, celui de Calabozo parlant même du «jougignominieuxaveclequella dynastiemalhonnêtedesMonagasa opprimélespueblosduVenezuela ${ }^{81}$. Maisleurobjectifestégalement de proclamer l'adhésion de ses signataires au processus entrepris par

78 Pronunciamiento de la Parroquia de Parapara, 30 de marzo de 1858. AGN/Caracas-Interior y Justicia. Tomo DCXVI, fol. 255.

79 Acta de pronunciamiento, Camaguán, 23 de marzo de 1858, San Fernando, Manuel María Betancourt impr. AGN/Caracas-Interior y Justicia. Tomo DCXVII, fol. 320.

80 Pronunciamiento de San Juan de Los Morros, 7 de marzo de 1858. AGN/Caracas-Interior y Justicia. Tomo DCXIX, fol. 296-296v. Ce thème est également présent dans le pronunciamiento de la ville de Calabozo daté du 23 mars. AGN/Caracas-Interior y Justicia. Tomo DCXVI, fol. 257.

81 Pronunciamiento de la Villa de Calabozo, 23 de marzo de 1858. AGN/Caracas-Interior y Justicia. Tomo DCXVI, fol. 257-258v. 
Julián Castro et ses partisans (inauguré par le pronunciamiento fondateur de Valencia).

Concernant leur rédaction, on peut observer qu'en dépit d'un plan, le contenu et les formes d'énonciation de ces pronunciamientos diffèrent. Ils commencent tous par reconnaître ce que Castro a entrepris, en viennent ensuite au récit des dénonciations et des méfaits causés par les Monagas à la nation, et se terminent par une série de points programmatiques qui comprennent la récusation de «l'autorité du Général J. T. Monagas comme Président de la République et desongendreOriach comme vice-président ${ }^{82}$, I'engagement à obéir aux nouvelles autorités centrales, provinciales et locales (dont certaines sont élues ou réélues dans le même temps que le pronunciamiento est rédigé) et au gouvernement provisoire, dans l'attente de la convocation d'une Grande Convention qu'ils appellent tous de leurs vœux. Ils prennent ainsi acte de la « dissolution du gouvernement » Monagas selon une logique qui n'est pas sans rappeler ce qui se produisit en 1808-1810 durant les «deuxannées cruciales ${ }^{83}$ consécutivesà l'invasion de la Péninsule, qui provoqua l'acéphalie du royaume après le renoncement au trône des Bourbons. En effet, 50 ans plus tard, les auteurs de ces pronunciamientos en appellent eux aussi à la légitime récupération, par chacun des corps constitués, de «sa souveraineté individuelle » comme l'écrivent de façon significative les membres de la paroisse de Zaraza ${ }^{84}$. Par le pronunciamiento, chacun contribue ainsi à sortir le pays de cet «état de désorganisation » et de « désunion », « de ce chaos » par le biais « d'un contrat partiel qui nous lie cependant au

82 Pronunciamiento de El Sombrero, 21 de marzo de 1858. AGN/Caracas-Interior y Justicia. Tomo DCXVI, fol. 267v.

83 En référence à l'article éponyme de GUERRA, François-Xavier. Dos años cruciales (1808-1809). In: GUERRA, François-Xavier. Modernidad e Independencias. Ensayos sobre las revoluciones hispánicas. Madrid: Editorial Mapfre, 1992, p.115-148.

84 Pronunciamiento de la parroquia de Zaraza, 23 de marzo de 1858. AGN/Caracas-Interior y Justicia. Tomo DCXVI, fol. 269v. 
reste de la République $»^{85}$, d'un «nouveau pacte fondamental ${ }^{86}$, dans l'attente d'élections libres qui permettront d'élire les représentants à la Convention. La notion de pacte/pactisme est bien toujours présente. Il faut comme au début du siècle, le dissoudre pour le refonder sur des bases nouvelles.

En miroir de ces actes d'adhésion et d'engagement ayant pour objectif de reconstituer, comme il est dit à plusieurs reprises, « un nouveau pacte social et un système véritablement démocratique », introduisant au passage une notion rarement employée dans un sens positif, on observe un glissement dans la dénonciation qui, au moment de refonder la République, vise de plus en plus ceux qui se refusent à rallier cette « révolution nationale » qui est celle du « peuple souverain ${ }^{87}$. C'est ainsi qu'on en appelle finalement à livrer combat à tous ceux qui s'opposent à suivre « cette juste cause », comme cela est très clairement affirmé dans le pronunciamiento de la ville de Chaguaramas:

"Tous les citoyens qui, de quelque façon que ce soit, se refusent à coopérer à la juste cause qui nous proclamons ici, seront considérés et traités comme ennemis de la Liberté et ne devront attendre pour cette même raison aucune considération car ils n'en sont pas dignes. ${ }^{88}$

Interroger selon cette dynamique, en prêtant attention à la construction narrative et au vocabulaire mobilisé, ces pronunciamientos nous renseignent l'incorporation de nouveaux concepts et sur la façon dont les acteurs se positionnent face aux institutions. C'est une

85 Ibidem.

86 Pronunciamiento de la parroquia de Parapara, 30 de marzo de 1858. AGN/Caracas-Interior y Justicia. Tomo DCXVI, fol. 255-255v. C'est moi qui souligne.

87 Pronunciamiento de San Sebastián, 18 de marzo de 1858. AGN/Caracas-Interior y Justicia. Tomo DCXIX, fol. 291.

88 Pronunciamiento de la Villa de Chaguaramas, 16 de marzo de 1858. AGN/Caracas-Interior y Justicia. Tomo DCXVI, fol. 274. 
autre façon de faire Etat qui émerge, certes parfois éloigné de ce que prescrivent les textes officiels, mais qui n'en démontrent pas moins qu'au-delà ou en marge des constitutions et institutions « de papier», d'autres répertoires del'action étatique sont inventés sur le terrain ou demandées par les acteurs locaux.

\section{Conclusion}

Ces deux campagnes, dont la physionomie et l'ampleur diffèrent, témoignent pourtant chacune à leur façon de ces formes alternatives d'interpellation mobilisation un large spectre d'acteurs, individuels et collectives. Plus encore, la lecture attentive de ces textes, au-delà de ce qui les motive, nous permet de scruter des formes de sociabilité mais aussi modes d'action et non pas uniquement de réaction. Prendre au mot l'invitation de M. D. Demélas, à savoir « dresser le tableau en ethnographe » de ces actes de rupture et reconstitution du pacte social afin de mettre au jour le pratiques qui adoptent parfois comme on vient de le voir des « formes théâtralisées, depuis l'organisation de la campagne avec le rassemblement des vecinos dans les lieux de sociabilités villageoises ou de la représentation municipale, en vue de rédiger le texte, d'en débattre, jusqu'à sa proclamation écrite, lue et célébrée avec ferveur et émotion ${ }^{89}$, $\mathrm{s}^{\prime}$ avère d'une grande pertinence. Cela permet dedémontrerque nousne sommes pas uniquement face à de simples actes de révolte ou de résistance, mais à des processus d'énonciationdecontre-hégémoniesquisupposentdesnégociations et qui produisent du politique. A tel point qu'on les retrouve de façon décuplée et reprise par une palette encore plus large d'acteurs à

89 DEMÉLAS-BOHY, Marie-Danielle. Le pronunciamiento : genèse d'une pratique. In : BERTRAND, Michel ; LAURENT, Natacha ; TAILLEFER, Michel (textes réunis par). Violences et pouvoirs politiques. Toulouse : Presses Universitaires du Mirail, 1996, p.90. Pour une mise au point sur ces questions, voir MISCHI, Julian. Introduction. Observer la politisation des ruraux sous l'angle des sociabilités : enjeux et perspectives. In : ANTOINE, Annie ; MISCHI, Julian (dir.). Sociabilité et politique en milieu rural. Rennes: PUR, 2008, p.7-21. 
la toute fin du siècle en réaction à la décision du président en place, le Général Guzmán Blanco à travers un texte célèbre de mai 1885 rédigé à Londres, de ne pas se présenter à sa succession pour les élections présidentielles qui doivent avoir lieu en 1886. Cette annonce donne lieu à la rédaction de plusieurs centaines de pronunciamientos (environ 400), dans le même temps que la presse et les corps constitués provinciaux et municipaux prennent également la plume. Plus encore, Fausto Teodoro de Aldrey, le propriétaire La Opinión Nacional (principal journal de l'époque), décide, avec une rapidité époustouflante au vu des moyens techniques de l'époque, de réunir ces centaines de documents dans un livre de plus de 1500 pages portant comme titre principal le même nom que celui retenu pour la campagne de 1828: El voto de Venezuela $a^{90}$... La façon dont il s'en explique dans l'introduction témoigne une fois encore de la légitimité très importante dont sont dotés ces écrits d'interpellation, qui participent pleinement du débat et parviennent à infléchir la dynamique politique. Enfin, l'inscription de cette pratique dans le paysage politique vénézuélien du XIXème permet également de mettre en évidence le rôle central joué par ces corps intermédiaires que sont les cabildos mais aussi les corps armés locaux pour traduire et proposer des compromis face aux injonctions

90 El Voto de Venezuela por el General Guzmán Blanco, ilustre americano, regenerador y pacificador de la República para la presidencia de la misma en el periodo constitucional de 1886-1888. Caracas: Imprenta de vapor de « la Opinión Nacional, 1885, 1424 p + un appendice. 
de l'Etat ou à une crise institutionnelle majeure, évitant ainsi des réponses violentes voire des soulèvements ${ }^{91}$.

\section{Sources et Bibliographie}

\section{Sources}

Archivo General de la Nacion/Caracas-Interior y Justicia, tomos DCXVI et DCXIX.

Coleccion de documentos relativos a la vida publica del Libertador de Colombia y del Peru Simon Bolivar. Para servir a la historia de independencia del Suramerica. Caracas: Imprenta de G. F. Devisme, 1828-18, Tomo decimotercio, 373p.

El Voto de Venezuela por el General Guzman Blanco, ilustre americano, regenerador y pacificador de la Republica para la presidencia de la misma en el periodo constitucional de 1886-1888. Caracas: Imprenta de vapor de « la Opinion Nacional, 1885, 1424 p + un appendice.

El Voto de Venezuela. O coleccion de actas y representaciones de las corporaciones civiles, militares y padres de familias de los departamentos de Venezuela, Maturin y Orenoco, dirigida a la Gran Convencion de Colombia y a SE el Libertador Presidente sobre reformas. Caracas: Devisme, $1829,315 p$.

VELASQUEZ, Ramon J. (ed.). Las Fuerzas armadas de Venezuela en el siglo

91 Comme l'a bien analysé Jean-Clément Martin pour la France révolutionnaire en opérant une analyse comparée entre opposant la Vendée et la Vallée du Rhône où l'absence de tiers entraîne des conflits violents et des massacres; au Sud-Ouest où les élites locales « anciennes ou nouvelles ont pu garder le contrôle de l'application des lois et ont ainsi éviter des soulèvements radicaux ». Jean-Clément MARTIN, « De la place des acteurs dans l'histoire », Revue d'histoire moderne et contemporaine, 49-4bis, supplément Histoire et anthropologie, nouvelles convergences ?, 2002, p. 94. 
XIX. Caracas : Presidencia de la Republica, 1971, 12 vols.

\section{Bibliographie}

ANNINO, Antonio. Pueblos, liberalismo y nacion en Mexico. In ANNINO, Antonio ; GUERRA, Francois-Xavier (dir.). Inventando la nacion: Iberoamerica siglo XIX. Mexico, Fondo de Cultura Economica, 2003, pp.399432.

BENOIT, Agnes. L'appel au pouvoir. Les petitions aux Parlements en France et au Royaume-Uni (1814-1848). Rennes: Presses Universitaires de Rennes, 2018

CALDERON, Maria Teresa. 1825-1832, Crisis y disolucion de la union colombiana. In: PORTILLO, Jose Maria ; CAGIAO VILA, Pilar (dir.). Entre imperio y naciones: Iberoamerica y el Caribe en torno a 1810. Santiago de Compostela: Universidad de Santiago de Compostela, 2012, p.215-244.

CHRISTIN, Olivier ; FOA, Jeremie (ed.). Petitions et suppliques, Annales de I'Est, Vol. 57, n. 2, 2007.

CONTAMIN, Jean-Gabriel. Contribution à une sociologie des usages pluriels des formes de mobilisation : I'exemple de la petition en France, these pour le doctorat en science politique, universite Paris 1, dir. Michel Offerle, 2001

DE LA FUENTE, Ariel. Facundo and Chaco in Songs and Stories: Oral Culture and the Representations of Caudillos in the Nineteenth-Century Argentine Interior. Hispanic American Historical Review, 80:3, 2000, p.503-535.

DEMELAS-BOHY, Marie-Danielle. Le pronunciamiento : genese d'une pratique. In : BERTRAND, Michel ; LAURENT, Natacha ; TAILLEFER, Michel (textes reunis par). Violences et pouvoirs politiques. Toulouse : Presses Universitaires du Mirail, 1996, p.73-92.

DEMELAS-BOHY, Marie-Danielle. Pactismo y constitucionalismo en los Andes. In. ANNINO, Antonio ; CASTRO LEIVA, Luis ; GUERRA, Francois-Xavier (dir.), De los Imperios a las Naciones : Iberoamerica. Zaragoza: IberCaja, 1994, p.495-510. Voir en particulier l'item intitule'El pronunciamiento como resurgimiento del pactismo', p.499-503.

DEMELAS, Marie-Danielle. Retour sur la pratique du pronunciamiento (1820-2013). Problemes d'Amerique latine, 2014/1, n. 91, p.41-51. 
DIONNET, Jean-Pierre. Le droit de petition durant la Restauration (18141830) : contribution à I'histoire socio-politique francaise du XIXeme siecle, these pour le doctorat en histoire du droit, universite de Poitiers, 2001

FALCON, Romana. El arte de la peticion: rituales de obediencia y negociacion, Mexico, segunda mitad del siglo XIX. Hispanic American Historical Review, vol. 86, n. 3, 2006, p.467-500.

FALLAS SANTANA, Carmen Maria. La voluntad de la Nacion y la regeneracion politica: Los pronunciamientos militares de 1859, 1868 y 1870 en Costa Rica, Dialogos Revista Electronica de Historia, Vol. 9, n. 2 Agosto 2008 - Febrero 2009, p.54-76.

FARGE, Arlette. Essai pour une histoire des voix au dix-huitieme siecle. Paris : Bayard editions, 2009.

GARCILAZO, Romina. Recurrir a las autoridades por una justa causa. El derecho de peticion en la ciudad de Rosario (Argentina), 1883/84-1890. Temas Americanistas, n. 33, 2014,pp.130-152.

GUERRA, Francois-Xavier. Dos anos cruciales (1808-1809). In: GUERRA, Francois-Xavier. Modernidad e Independencias. Ensayos sobre las revoluciones hispanicas. Madrid: Editorial Mapfre, 1992, p.115-148.

GUERRA, Francois-Xavier. El pronunciamiento en Mexico: practicas e imaginarios. Trace, n. 37, juin 2000, pp.15-26.

HEBRARD, Veronique. Dinamicas de movilizacion y logicas de socializacion de lo politico en el mundo rural venezolano, 1858-1859. Illes i imperis, n. 17, 2015, p.113-136.

HEBRARD, Veronique. El recurso al poder personal y autoritario ». In HEBRARD, Veronique. La Venezuela independiente. Una nacion de discurso (1808-1830). Madrid: Vervuert/lberoamicana, 2012, p.446-548.

HEBRARD, Veronique. Opinion publique et representation dans le Congres Constituant venezuelien (1810-1812). Annales Historiques de la Revolution Francaise, n. 365 " Lumieres et revolutions en Amerique latine », Juillet-septembre 2011, p.153-175.

HEBRARD, Veronique. Quand la parole est collective. In. Veronique HEBRARD, La Faction de la Sierra. Un apprentissage du politique entre engagement et contrainte.Venezuela, 1858-1859. Inedit d'HDR.Universite Paris Ouest Nanterre La Defense, 2013, p.249-255. HAL Id:tel-01180313, version 1. 
IRWIN, Domingo ; MICETT, Ingrid. De caudillos a pretorianos. Una Periodizacion de la realidad militar venezonala, siglos XIX-XX. Nuevo Mundo Mundos Nuevos [En linea], Cuestiones del tiempo presente, Puesto en linea el 31 enero 2011. DOI : 104000/nuevomundo.60783.

MEROT, Catherine (dir.). L'individu face au pouvoir : les petitions aux assemblees parlementaires. Revue administrative, 61 eme annee, numero special, 2008.

MISCHI, Julian. Introduction. Observer la politisation des ruraux sous I'angle des sociabilites : enjeux et perspectives. In : ANTOINE, Annie ; MISCHI, Julian (dir.). Sociabilite et politique en milieu rural. Rennes : PUR, 2008, p.7-21.

REBERIOUX, Madeleine. Petitionner ». In : REBERIOUX, Madeleine (dir.). Pour que vive I'histoire. Ecrits. Paris : Belin, 2017. pp.449-455.

THIBAUD, Clement. Entre les cites et l'Etat. Caudillos et pronunciamientos en Grande-Colombie. Geneses, 2006/1, n. 62, p.5-26.

VERDO, Genevieve. Pierre Rosanvallon, archeologue de la democratie. Revue historique, 2002, n. 3, pp.693-720.

WAHNICH, Sophie. La petition : une politisation de la plainte, 1789-1792. Annales de l'Est, 2007-2. pp.71-87. 